\title{
Chemical Warfare Agent Detection in Complex Environments with Comprehensive Two-Dimensional Gas Chromatography
}

\author{
Stephen E. Reichenbach ${ }^{a}$, Mingtian $\mathrm{Ni}^{a}$, Visweswara Kottapalli ${ }^{a}$, Arvind Visvanathan ${ }^{a}$, \\ Edward B. Ledford, Jr. ${ }^{b}$, John Oostdijk ${ }^{c}$, and Henk C. Trap ${ }^{c}$ \\ ${ }^{a}$ Computer Science \& Engineering Dept., University of Nebraska, Lincoln NE 68588 USA \\ ${ }^{b}$ Zoex Corporation, 2611 West M St, Suite D, Lincoln NE 68522 USA \\ ${ }^{c}$ TNO - Prins Maurits Laboratory, PO 45, 2280 AA, Rijswijk (ZH) Netherlands
}

\begin{abstract}
Comprehensive two-dimensional gas chromatography (GCxGC) is an emerging technology for chemical separation that provides an order-of-magnitude increase in separation capacity over traditional gas chromatography. GCxGC separates chemical species with two capillary columns interfaced by two-stage thermal desorption. Because GCxGC is comprehensive and has high separation capacity, it can perform multiple traditional analytical methods with a single analysis. GCxGC has great potential for a wide variety of environmental sensing applications, including detection of chemical warfare agents (CWA) and other harmful chemicals.

This paper demonstrates separation of nerve agents sarin and soman from a matrix of gasoline and diesel fuel. Using a combination of an initial column separating on the basis of boiling point and a second column separating on the basis of polarity, GCxGC clearly separates the nerve agents from the thousands of other chemicals in the sample. The GCxGC data is visualized, processed, and analyzed as a two-dimensional digital image using a software system for GCxGC image processing developed at the University of Nebraska - Lincoln.
\end{abstract}

Keywords: chemical warfare agents, CWA, comprehensive two-dimensional gas chromatography, GCxGC, chemical separations, chemical sensing, chemical detection, sarin, soman

\section{INTRODUCTION}

Since the early 1960's, the predominant method for detecting chemical warfare agents (CWA) has been gas chromatography (GC). ${ }^{1}$ Although most CWA are suited for GC analysis, there is no single, universal GC method for detecting all CWA, nor even a majority of them. In present-day practice, different analytical methods and instruments are required for different classes of CWA (nerve agents, vesicants, irritants, and potential CW toxins), and for the different matrices (air, water, soil, vegetation, food, and biological fluids) in which they are found. This variety of methods and equipment necessitates well-equipped laboratories staffed with expert personnel, as well as extensive safety precautions and logistics.

There are other problems, beyond the high methodological and personnel overhead, with CWA detection using current GC technology. A major problem with present-day detection technology is the false positive rate, caused by environmental interferences, especially from hydrocarbon background. In the case of air sampling, single analytical methods for detecting the majority of CWA exist, ${ }^{2}$ but suffer significant drawbacks, including moderate sensitivity, analysis times in the range of tens of minutes, and lack of automation. In practice, these drawbacks make real-time field monitoring of CWA with current analytical methods difficult, and often impossible.

In view of recent world events, a universal, sensitive, rapid, and automated GC method for detecting CWA in air is desirable for time-critical atmospheric monitoring of both indoor and outdoor environments. The Committee on R\&D Needs for Improving Civilian Medical Response to Chemical and Biological Terrorism Incidents noted that "the civilian medical community must find ways to adapt the many new and emerging

Additional author information: S. Reichenbach (corresponding author), reich@cse.unl.edu, Phone 1.402.472.5007. M. Ni, mni@cse.unl.edu. V. Kottapalli, viskott@cse.unl.edu. A. Visvanathan, avisvan@cse.unl.edu. E. Ledford, ed@zoex.com, Phone 1.402.475.7640. J. Oostdijk, oostdijk@pml.tno.nl, Phone 31-(0)15-284-3077. H. Trap, trap@pml.tno.nl, Phone 31(0)15-284-3481. 
detection technologies to the spectrum of chemical and biological warfare agents" and specifically recommended research and development of gas chromatography technology. ${ }^{3}$

An opportunity to meet these technical challenges exists in the form of a new gas chromatographic technique known as comprehensive two-dimensional gas chromatography (GCxGC). GCxGC has established that the hydrocarbon background consists of thousands of chemical species, ${ }^{4}$ a level of complexity that makes one-dimensional chemical separation techniques problematical with regard to CWA detection. As demonstrated in this paper, GCxGC effectively separates CWA from complex hydrocarbon background. Comprehensive two-dimensional separation promises to be an essential component of viable CWA sensing systems.

Section 2 outlines the GCxGC separation process and computer processing of GCxGC data. Section 3 demonstrates GCxGC detection of nerve agents sarin and soman in a mixture of gasoline and diesel fuel. Section 4 briefly considers some research issues for CWA detection with GCxGC.

\section{BACKGROUND}

\subsection{Comprehensive two-dimensional gas chromatography}

Comprehensive two-dimensional gas chromatography (GCxGC) is an emerging technology for chemical separation that provides an order-of-magnitude increase in separation capacity over traditional GC. ${ }^{5}$ GCxGC separates chemical species with two capillary columns interfaced by two-stage thermal desorption. ${ }^{6,7}$ Figure 1 illustrates the system components for comprehensive GCxGC with thermal modulation.

As shown in Figure 2, the GCxGC output can be displayed as an image, with pixels arranged so that the abscissa (X-axis, left-to-right) is the elapsed time for the first column separation and the ordinate (Y-axis, bottom-to-top) is the elapsed time for the secondary column separation. Each pixel value indicates the rate at which molecules are detected at a specific time. Each resolved chemical substance in a sample produces a small blob, i.e., a peak or cluster of pixels, with values that are larger than the background values. In Figure 2, the smaller values of the background are colorized light blue and the larger values of the blob peaks are colorized with dark blue, magenta, and white.

GCxGC offers significantly greater separation capacity than traditional one-dimensional GC. Figure 3 illustrates the one-dimensional chromatogram for the sample in Figure 2 (response as a function of time, equivalent to the sums of all pixels in each vertical column of the image). As seen in Figure 2, GCxGC separates thousands of different chemicals, whereas it is difficult to distinguish even a few hundred peaks in the data shown in Figure 3. Peaks that are clearly separated in GCxGC co-elute in a one-dimensional chromatogram, interfering with accurate quantification. Due to the extreme toxicity of nerve agents (less than $10 \mu \mathrm{g} / \mathrm{kg}$ ), CWA detection requires discrimination of even small peaks in the presence of an overwhelming matrix.

Peak patterns that are clearly visible in GCxGC images are chemically significant and so offer important additional information. Structural regularities among analytes give rise to patterns in the distribution of GCxGC

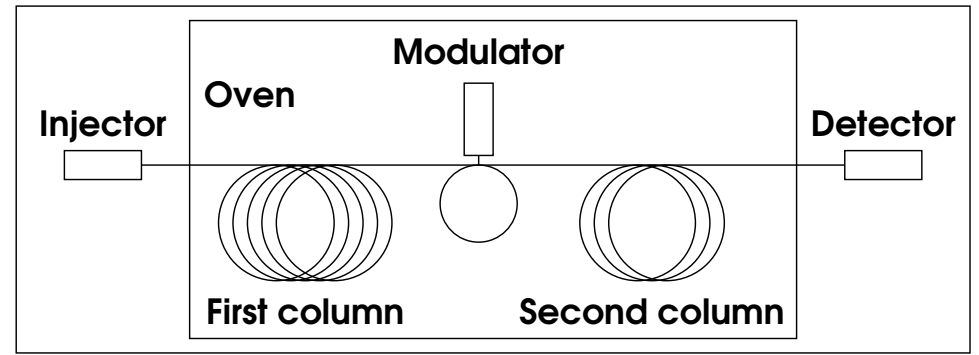

Gas Chromatograph

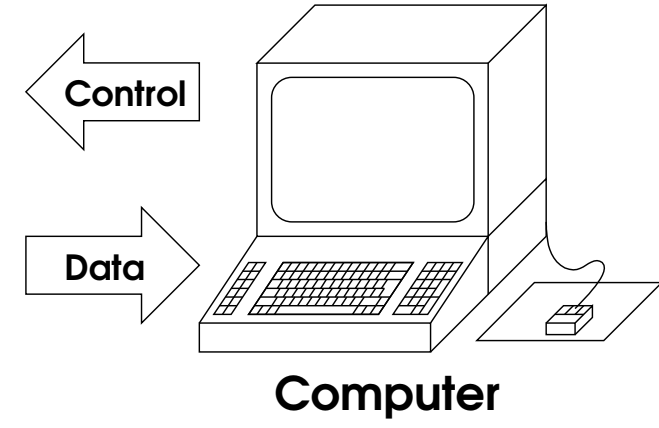

Figure 1. Instrumentation for comprehensive two-dimensional gas chromatography (GCxGC) with thermal modulation. 


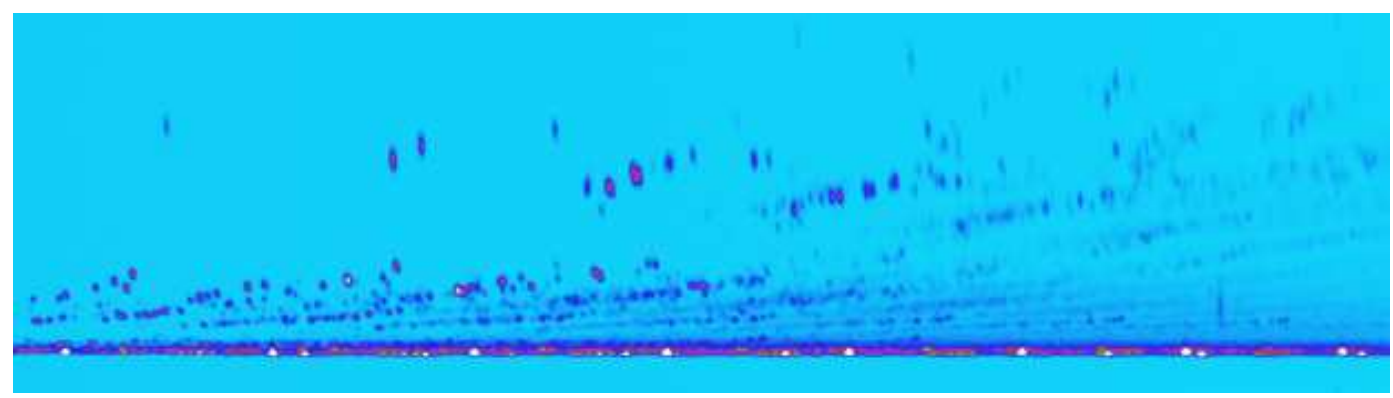

Figure 2. A GCxGC image of diesel oil (Zoex Corporation ${ }^{8}$ ).

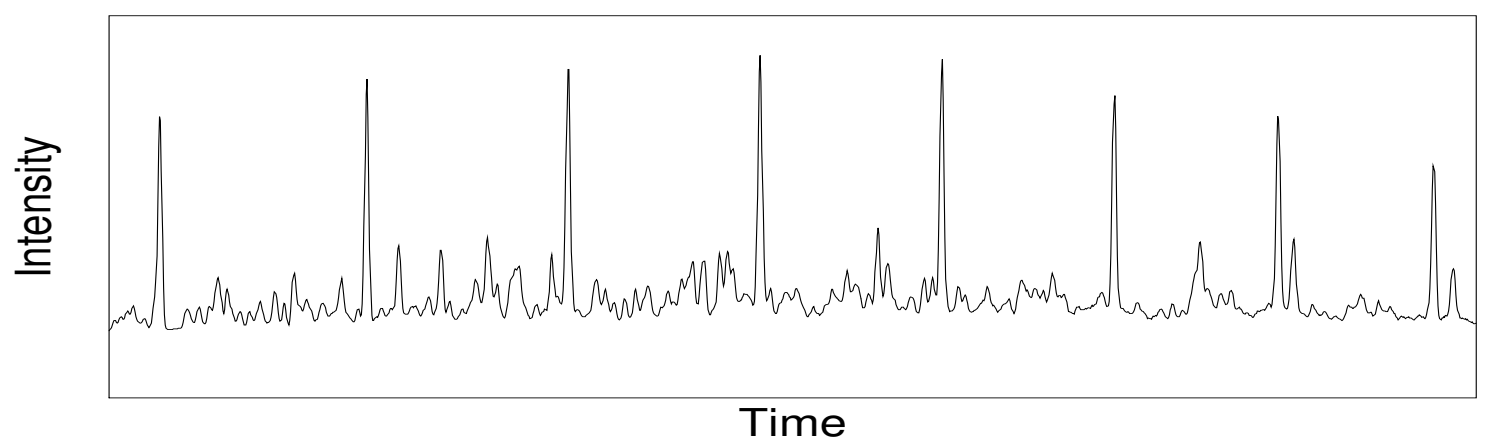

Figure 3. Traditional one-dimensional gas chromatogram corresponding to the image in Figure 2.

peaks. Petroleum liquids, or the hydrocarbon background of air, exhibit nominally horizontal bands in the GCxGC image, corresponding to chemical classes, within which isomers form diagonal sub-bands. In Figure 2, the aliphatic hydrocarbons form a band near the bottom of the image, within which the n-alkanes produce prominent peaks at nearly regular intervals. Other chemical classes, such as the single-ring and double-ring aromatics are located above the band of aliphatic hydrocarbons in Figure 2.

The increased separation bandwidth of GCxGC holds great promise for many important applications, including CWA detection. In general, the upper-left corner of the retention plane tends to be unpopulated by hydrocarbon species. The reason for this is that the first column separates on the basis of boiling point, the second on the basis of polarity. Light hydrocarbons, which elute early on the first dimension, are not polar enough to exhibit long retention times in the second dimension. This creates a void in the retention plane above the light aromatics. CWA agents, which are considerably more polar than hydrocarbons of comparable boiling point, tend to populate the otherwise empty region of hydrocarbon chromatograms. The ability of GCxGC to separate nerve agents from hydrocarbon background could form the basis of a sensor with low false positive rates.

With its greatly increased performance, GCxGC generates data in significantly larger quantity and with significantly greater complexity than traditional GC. Compared to traditional GC data, the GCxGC image has hundreds of times more data points, an order-of-magnitude more distinct peaks, and richer positional relationships (patterns) between peaks. The quantity and complexity of GCxGC data makes human analyses of GCxGC images difficult and time-consuming and motivates the need for computer processing.

\subsection{GCxGC Image Processing and Analysis}

The goal of GCxGC analyses is to separate, quantify, and identify specific chemicals in a given sample. The principal image processing and analysis tasks are: 
- Calibration and correction of the acquisition system (e.g., removing baseline offset and determining chemical response factors).

- Segmentation of the image into individual blobs and background.

- Quantitative measurement of each blob of interest.

- Chemical identification of each blob of interest.

The output of this process should be an accurate and precise listing by name of the chemical components and the quantity of each.

GC Image ${ }^{T M}$ is a software system developed at the University of Nebraska - Lincoln for visualizing, processing, analyzing, and reporting on GCxGC chemical separations. ${ }^{9}$ The software utilizes a powerful graphical userinterface (GUI) that enables users to:

- Import and visualize GCxGC image data.

- Detect and correct acquisition artifacts.

- Detect, edit, and quantify blobs of pixels associated with chemical peaks.

- Define, edit, and recognize patterns of chemical peaks.

- Generate reports characterizing selected chemical peaks.

The following example illustrates some of these operations on a calibration mixture containing four n-alkanes, dimethylbenzene, sarin, and soman, illustrated in Figure 4. The basic operations are:

1. Import the data.

2. Remove the baseline offset.

3. Colorize the data for visualization.

4. Detect, quantify, and identify chemical compounds associated with blob peaks.

5. Report results.

The calibration mixture was used to establish retention-time windows under the run conditions described in Section 3.

The computer software must convert raw GCxGC data to an image format for visualization and processing. For example, the raw data acquired as described in Section 3 is imported from a file in comma-separated-value (CSV) format and stored as a two-dimensional digital image for subsequent processing.

The raw data exhibits a slowly varying, non-zero baseline, due primarily to detector offset and secondary column bleed. The baseline is about 18 picoamps at the beginning of the data, falls to about 16.5 picoamps in the middle of the data, and then rises to about 19.5 picoamps at the end of the data. The baseline level can be determined from the background values and removed, ${ }^{10}$ yielding corrected data in which the baseline is nearly uniformly zero across the data.

GCxGC data can be visualized in a variety of ways - as a two-dimensional image, as an array of numerals, as one-dimensional slices and projections, or as a surface with three-dimensional perspective viewing. In Figure 4, the image for the CWA calibration run is colorized using a scale from light-blue for small values; through dark blue, green, brown, and red; to white for large values.

Blob detection is the process of separating and quantifying contiguous groups of data values comprising a peak. Figure 4 indicates eight detected peaks. The graphical overlay in Figure 4 shows dimethylbenzene as a marker peak connected to the four n-alkane peaks below, sarin to the upper-left, and soman isomers to the upper-right. The isomers m-dimethylbenzene and p-dimethylbenzene co-elute in a single peak. The two peaks 


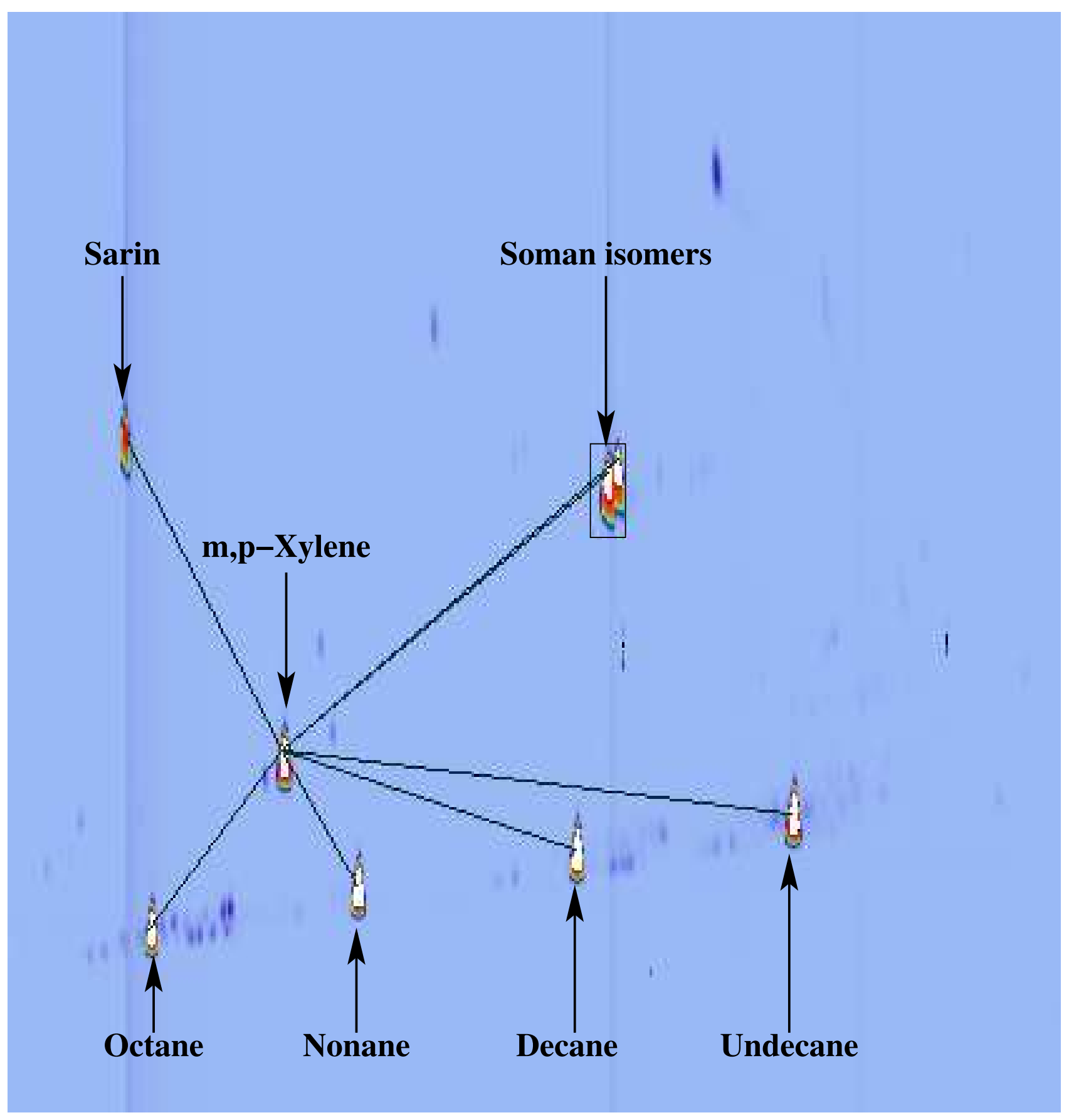

Figure 4. A calibration mixture of sarin, soman, and hydrocarbon standards analyzed by GCxGC and rendered as a colorized image. 
for soman are characteristic and result from stereoisomerism. This nerve agent consists of four stereoisomers due to chirality at pinacolyl moiety and at phosphorous. Two epimeric pairs of enantiomers of soman are separated, with pair $\mathrm{C}(+) \mathrm{P}(-)$-soman and $\mathrm{C}(-) \mathrm{P}(+)$-soman on the left and pair $\mathrm{C}(+) \mathrm{P}(+)$-soman and $\mathrm{C}(-) \mathrm{P}(-)$-soman on the right ( $\mathrm{C}$ for chirality in the pinacolyl moiety and $\mathrm{P}$ for chirality at phosphorus).

The pattern of blob peaks in one image can be used as a template for automatically recognizing the same chemicals in subsequent images. For example, the chemical names of the blobs detected in the calibration mixture can be identified interactively by name. Then, the pattern of chemicals observed and interactively identified in the calibration mixture can be saved to a template file and used to automatically recognize and name those chemicals in subsequent sample runs. The graphical overlay in Figure 4 indicates the chemical names identified with each peak and the template that can be used for recognizing the peak pattern in subsequent images.

Table 1 lists the detected chemicals, retention times, and integrated blob peak values in the calibration mixture. The integrated blob peak values are computed as the sum of detector response values (in pico amps, after background removal) within the extent of the blob.

\begin{tabular}{lccc}
\hline Chemical Name & \multicolumn{2}{c}{ Retention Time } \\
& $\begin{array}{c}\text { Column 1 } \\
\text { (minutes) }\end{array}$ & $\begin{array}{c}\text { Column 2 } \\
\text { (seconds) }\end{array}$ & Peak Integration \\
(pico amps)
\end{tabular}

Table 1. Retention times and integrated peak values of chemicals in the calibration mixture.

\section{EXPERIMENTAL RESULTS}

CWA detection was performed by GCxGC at the TNO - Prins Maurits Laboratory, using a first column $15.0 \mathrm{~m}$ in length with a $250 \mu \mathrm{m}$ inside diameter and a DB-1 stationary phase $1.0 \mu \mathrm{m}$ thick. The thermal modulator, manufactured by Zoex Corporation (Lincoln NE), employs a column loop cooled by a liquid nitrogen gas stream and heated by a pulsed hot jet, with 2 second thermal modulation cycle. The modulator tube consisted of uncoated deactivated fused silica tubing with inside diameter of $100 \mu \mathrm{m}$. The second dimension column was $1.0 \mathrm{~m}$ in length with $100 \mu \mathrm{m}$ inside diameter, coated with DB-WAX (J\&W Corporation) $0.1 \mu \mathrm{m}$ thick. (Other second dimension columns were tested, but are not reported here, including uncoated fused silica, $3.8 \mathrm{~cm} \mathrm{DB}$ wax column with $100 \mu \mathrm{m}$ and $0.1 \mu \mathrm{m}$ film thickness, and $1 \mathrm{~m}$ CP-Sil 88 column similar to BPX-50 with $100 \mu \mathrm{m}$ and $0.1 \mu \mathrm{m}$ film thickness.) The injector was an Optic III large volume injection system (Atas, Veldhoven, The Netherlands) operated in split mode at $260 \mathrm{C}$, split ratio 20 , and injection volume $1.0 \mu \mathrm{l}$. Column head pressure was $38 \mathrm{psi}(264 \mathrm{kPa})$. The gas chromatograph was an Agilent 6890 equipped with standard flame ionization detector. The $\mathrm{GC}$ oven was ramped from $40 \mathrm{C}$ to $240 \mathrm{C}$ at a rate of $10 \mathrm{C} / \mathrm{min}$, resulting in a 20 minute run, sampled at $200 \mathrm{~Hz}$.

The test mixture was a 1:1 mix by volume of Shell gasoline and diesel fuel, spiked with nerve agents sarin and soman at concentrations high enough to permit their clear distinction. Figure $\mathbf{5}$ illustrates the data after baseline correction and colorization. Both Figures 4 and 5, show the last 14 minutes of the run, which contain the CWA and hydrocarbons of interest. (The first 6 minutes of the runs, which contain solvent front, are not shown.) The pattern of chemicals in the calibration mixture illustrated in Figure 4 is clearly visible in Figure 5 and can be automatically recognized. Other hydrocarbon chemicals and chemical groups also can be identified. ${ }^{4}$ Table 2 lists some of the chemicals present, retention times, and (uncalibrated) integrated blob peak values. No 


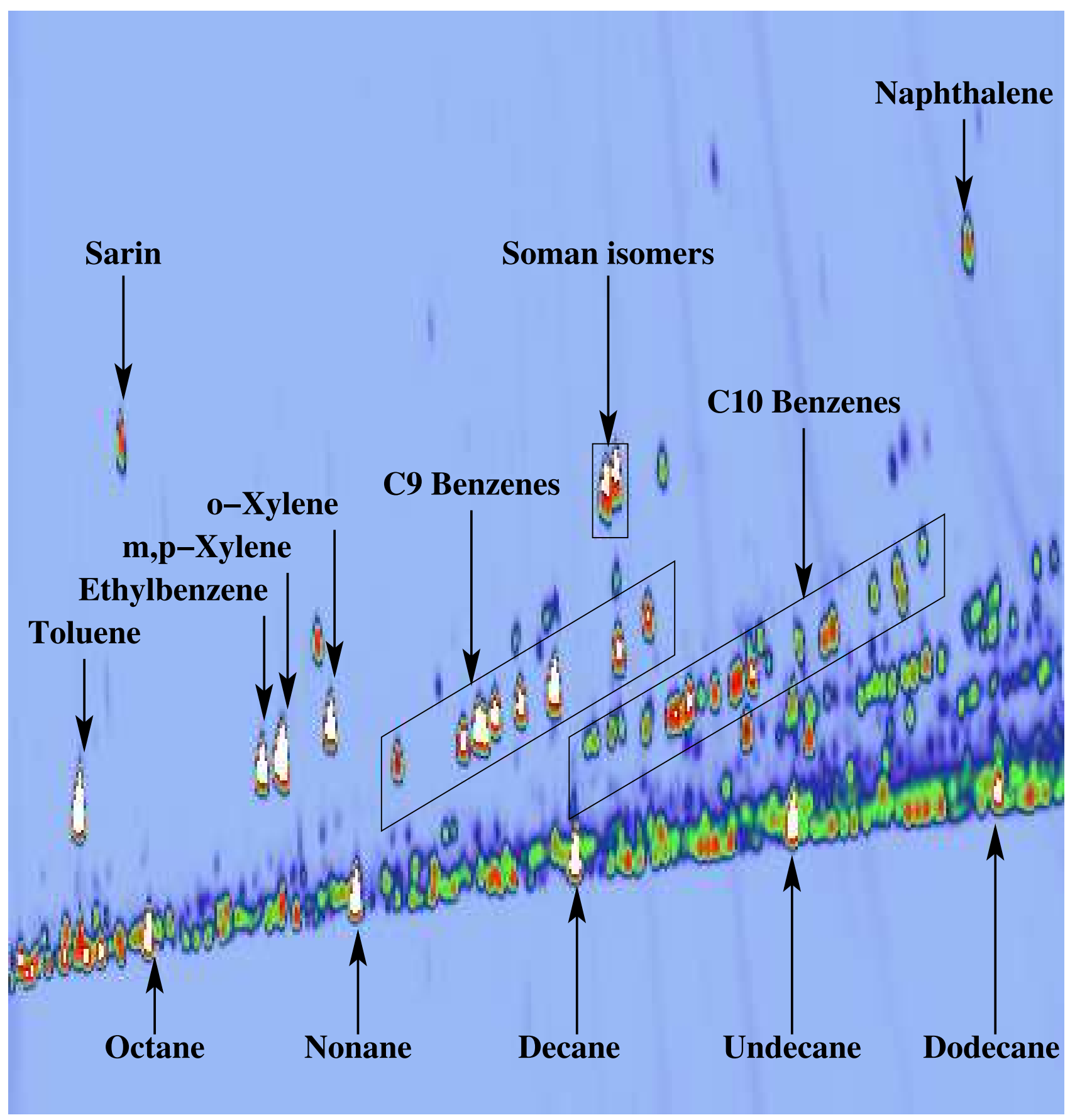

Figure 5. A test mixture with sarin, soman, gasoline, and diesel fuel analyzed by GCxGC and rendered as a colorized image. 


\begin{tabular}{|c|c|c|c|}
\hline \multirow[t]{2}{*}{ Chemical Name } & \multicolumn{2}{|c|}{ Retention Time } & \multirow{2}{*}{$\begin{array}{l}\text { Peak Integration } \\
\text { (pico amps) }\end{array}$} \\
\hline & $\begin{array}{l}\text { Column } 1 \\
\text { (minutes) }\end{array}$ & $\begin{array}{r}\text { Column } 2 \\
\text { (seconds) }\end{array}$ & \\
\hline decane & 13.50 & 0.48 & 650,386 \\
\hline dodecane & 19.10 & 0.59 & 224,768 \\
\hline ethylbenzene & 9.37 & 0.64 & 517,141 \\
\hline 2-ethyltoluene & 12.80 & 0.75 & 146,210 \\
\hline 3-ethyltoluene/4-ethyltoluene & 12.23 & 0.72 & 568,738 \\
\hline indan & 14.50 & 0.90 & 65,492 \\
\hline isopropylbenzene & 11.13 & 0.64 & 48,612 \\
\hline m-xylene/p-xylene & 9.63 & 0.68 & $1,547,572$ \\
\hline naphtalene & 18.73 & 1.58 & 38,869 \\
\hline nonane & 10.60 & 0.41 & 600,204 \\
\hline o-xylene & 10.27 & 0.72 & 400,391 \\
\hline octane & 7.83 & 0.33 & 546,939 \\
\hline propylbenzene & 12.00 & 0.68 & 119,532 \\
\hline sarin & 7.50 & 1.24 & 39,729 \\
\hline soman epimers & 13.93 & 1.16 & 204,950 \\
\hline soman epimers & 14.07 & 1.19 & 158,040 \\
\hline toluene & 6.93 & 0.58 & $2,093,891$ \\
\hline 1,2,3-trimethylbenzene & 14.10 & 0.84 & 115,861 \\
\hline 1,2,4-trimethlybenzene & 13.27 & 0.80 & 587,092 \\
\hline 1,3,5-trimethlybenzene & 12.43 & 0.73 & 157,714 \\
\hline undecane & 16.40 & 0.55 & 700,330 \\
\hline
\end{tabular}

Table 2. Retention times and integrated peak values of some chemicals in the test mixture.

calibrated quantitative results were derived from these experiments. However, given the clear separation of the nerve agents from the hydrocarbon matrix and the focusing associated with thermal modulation, the detection limits in GCxGC should be significantly improved with respect to conventional GC.

\section{CONCLUSION}

GCxGC provides a clear separation of nerve agents sarin and soman from hydrocarbon background. This improved separation, as compared to conventional GC, suggests that GCxGC is the basis for improved CWA detection, with lower detection limits and reduced false positive rates. Among the issues that remain to be investigated are:

- quantitative analysis of CWA by GCxGC,

- the applicability of GCxGC to other CWA,

- interferences from heteroatomic compounds such as common cleaning agents, and

- the efficacy of mass spectromety with GCxGC for confirmatory CWA analyses.

Gas-chromatography with mass-spectrometry (GC-MS) has been used for CWA detection, ${ }^{11,12}$ including mobile GC-MS instruments for near-real-time CWA detection. ${ }^{13}$ One of the problems with GC-MS is the unmixing of a mass spectrum for co-eluted analytes. ${ }^{14,15}$ This problem is greatly reduced in GCxGC.

GCxGC provides greater separation capacity than GC and so is even better suited for a pairing with MS. Time-of-Flight MS (TOFMS) is capable of the rapid acquisition required by GCxGC. GCxGC is important for MS because the better separations significantly reduce co-elution and the problems of mass spectrum unmixing. And, MS is important for GCxGC because GCxGC data is far richer (and hence more complex) than GC separations. In particular, MS holds great promise as a tool for computer-assisted and automated analyses of compounds separated by GCxGC. 


\section{ACKNOWLEDGMENTS}

This research was supported by the U.S. National Science Foundation, Award 0231746.

\section{REFERENCES}

1. Z. Witkiewicz, M. Mazurek, and J. Szulc, "Chromatographic analysis of chemical warfare agents," Journal of Chromatography A 503, pp. 293-357, 1990.

2. W. Carrick, D. Cooper, and B. Muir, "Retrospective identification of chemical warfare agents by hightemperature automatic thermal desorption-gas chromatography-mass spectrometry," Journal of Chromatography A 925, pp. 241-249, 2001.

3. Committee on R\&D Needs for Improving Civilian Medical Response to Chemical and Biological Terrorism Incidents, Chemical and Biological Terrorism: Research and Development to Improve Civilian Medical Response, National Academy Press, Washington DC, 1999.

4. G. S. Frysinger, R. B. Gaines, and E. B. Ledford, Jr., "Quantitative determination of BTEX and total aromatic compounds in gasoline by comprehensive two-dimensional gas chromatography (GCxGC)," Journal of High Resolution Chromatography 22(4), pp. 195-200, 1999.

5. W. Bertsch, "Two-dimensional gas chromatography. concepts, instrumentation, and applications - Part 2: Comprehensive two-dimensional gas chromatography," Journal of High Resolution Chromatography 23(3), pp. 167-181, 2000.

6. E. B. Ledford, Jr. and C. A. Billesbach, "Jet-cooled thermal modulator for comprehensive multidimensional gas chromatography," Journal of High Resolution Chromatography 23(3), pp. 202-204, 2000.

7. J. Beens, M. Adahchour, R. J. Vreuls, K. van Altena, and U. A. Brinkman, "Simple, non-moving modulation interface for comprehensive two-dimensional gas chromatography," Journal of Chromatography A 919(1), pp. 127-132, 2001.

8. E. B. Ledford, Jr., Zoex Corp., 2002.

9. S. E. Reichenbach, M. Ni, V. Kottapalli, A. Visvanathan, and E. B. Ledford, Jr., "Information technologies for comprehensive two-dimensional gas chromatography," in International Symposium on Capillary Chromatography, p. CDROM:to appear, 2003.

10. S. E. Reichenbach, M. Ni, D. Zhang, and E. B. Ledford, Jr., "Image background removal in comprehensive two-dimensional gas chromatography," Journal of Chromatography A 985(1), pp. 47-56, 2003. A preliminary copy of this paper appeared in International Symposium on Capillary Chromatography, CDROM:11p, 2002.

11. J. Steinhanses and K. Schonene, "Thermal desorption-gas chromatography of some organophosphates and s-mustard after trapping on tenax," Journal of Chromatography A 514, pp. 273-278, 1990.

12. C. E. Kientz, "Chromatography and mass spectrometry of chemical warfare agents, toxins and related compounds: State of the art and future prospects," Journal of Chromatography A 814, pp. 1-23, 1998.

13. J. Stuff, R. Cheicante, D. D. H., and J. Ruth, "Detection of the chemical warfare agents bis- $(2-$ chloroethyl)ethylamine (hn-1) and tris-(2-chloroethyl)amine (hn-3) in air," Journal of Chromatography A 849, pp. 529-540, 1999.

14. J. W. Biller and K. Biemann, "Reconstructed mass spectra: A novel approach for the utilization of gas chromatographic data," Anal. Lett. 7, pp. 515-528, 1974.

15. B. N. Colby, "Spectral deconvolution of overlapping GC/MS components," J. Am. Soc. Mass Spectrom. 3(5), pp. 558-562, 1992. 